\title{
Rotational excitation of sulfur monoxide in collision with helium at high temperature
}

\author{
F. Lique, M.-L. Dubernet, A. Spielfiedel, and N. Feautrier
}

LERMA and UMR 8112 of CNRS, Observatoire de Paris-Meudon, 92195 Meudon Cedex, France

e-mail: francois.lique@obspm.fr

Received 15 November 2005 / Accepted 15 December 2005

\section{ABSTRACT}

Context. Over the next few years, ALMA and Herschel missions will open the universe to high spatial and spectral resolution studies at infrared and sub-millimeter wavelengths. This will allow investigators to study in much greater detail the composition and evolution of molecules in space. Modeling of the spectra will require accurate radiative and collisional rates of species of astrophysical interest.

Aims. We calculate new rates coefficients for the 91 first rotational levels of the SO molecule in collision with He for temperatures ranging from 50 to $300 \mathrm{~K}$.

Methods. Different levels of approximations (IOS, IOS scaling relationship) are tested in comparison with the exact full close coupling approach. From the analysis of the validity range of each method, we define a hybrid approach combining the accuracy of the full close coupling method and the less time consuming approximations (IOS, IOS scaling relationship).

Results. The new rate coefficients are calculated up to $300 \mathrm{~K}$. These coefficients differ significantly from previously published ones.

Key words. ISM: molecules - molecular processes - molecular data

\section{Introduction}

Observations of molecular emission at millimeter and infrared wavelengths, supplemented by careful and detailed modeling, are powerful tools to investigate the physical and chemical conditions of astrophysical objects. First observed by Gottlieb \& Ball (1973) in the interstellar gas, sulfur monoxide has been used extensively to study shocked molecular gas in various environments (Turner et al. 1992; Van Dishoeck et al. 1998; Blake et al. 1987). SO is a good probe since the fine structure in its ${ }^{3} \Sigma^{-}$electronic ground state gives many relatively close transitions. The modeling of the line intensities requires comparison with model excitation calculations using radiative as well as collisional rates. It is thus of a great importance to provide accurate collisional rates for a large temperature range. Collisional excitation in the interstellar medium is dominated by collisions with the most abundant neutral species, $\mathrm{He}$ and $\mathrm{H}_{2}$.

Excitation rates by collision among fine structure levels of $\mathrm{SO}$ with $\mathrm{H}_{2}$ were provided some time ago by Green (1994), using coupled states and recoupling approximations of collision dynamics and the potential energy surface (PES) obtained for $\mathrm{CS}-\mathrm{H}_{2}$ with an electron-gas model (Green \& Chapman 1978). Spin-free rates were obtained for temperatures from 50 to $350 \mathrm{~K}$ and rates of fine structure levels were obtained from the spin-free rates using the recoupling method described by Neufeld \& Green (1994). In Paper I (Lique et al. 2005), we calculated collisional rates among the 31 first fine structure levels of SO for temperatures ranging from 5 to $50 \mathrm{~K}$. The dynamics calculations were performed using a new accurate potential energy surface for the SO-He system. The full close coupling approach was used for the dynamics. The tested approximate approaches are not accurate for calculations at low temperatures involving low energy levels where the fine structure splitting is large compared to the rotational splitting. However, it was shown that the recoupling technique from IOS cross sections should provide a reasonable estimate of cross sections for high- $N$ levels and at large energies.

As the number of open rotational channels increases rapidly with energy, we propose here a hybrid approach combining full close coupling for the first levels and different levels of approximations for the higher levels and at high energy. In the next section, we briefly describe the computation methodology, including close coupling, infinite order sudden (IOS) and IOS scaling relationships (hereafter IOSR) scattering techniques. A comparison between these different approaches is discussed in Sect. 3, and results are given in Sect. 4 where a comparison with earlier calculations is also provided. Section 5 summarizes the conclusions.

\section{Computation methodology}

The PES was calculated at the RCCSD(T) level (Knowles et al. 1993, 2000). The SO $r$-distance was fixed at its experimental 
Table 1. Energy in $\mathrm{cm}^{-1}$ of the fine structure levels.

\begin{tabular}{|c|c|c|c|c|c|c|c|c|c|c|c|}
\hline Level & $N$ & $j$ & Energy & Level & $N$ & $j$ & Energy & Level & $N$ & $j$ & Energy \\
\hline 1 & 1 & 0 & 0.000 & 32 & 11 & 12 & 97.963 & 63 & 21 & 20 & 335.742 \\
\hline 2 & 0 & 1 & 1.001 & 33 & 11 & 10 & 99.302 & 64 & 21 & 21 & 340.566 \\
\hline 3 & 1 & 2 & 3.100 & 34 & 11 & 11 & 103.865 & 65 & 22 & 23 & 366.396 \\
\hline 4 & 2 & 3 & 6.412 & 35 & 12 & 13 & 115.221 & 66 & 22 & 21 & 367.272 \\
\hline 5 & 1 & 1 & 10.552 & 36 & 12 & 11 & 116.746 & 67 & 22 & 22 & 372.107 \\
\hline 6 & 2 & 1 & 10.987 & 37 & 12 & 12 & 121.088 & 68 & 23 & 24 & 399.372 \\
\hline 7 & 3 & 4 & 11.021 & 38 & 13 & 14 & 133.906 & 69 & 23 & 22 & 400.234 \\
\hline 8 & 2 & 2 & 13.424 & 39 & 13 & 12 & 135.091 & 70 & 23 & 23 & 405.078 \\
\hline 9 & 3 & 2 & 14.632 & 40 & 13 & 13 & 139.745 & 71 & 24 & 25 & 433.777 \\
\hline 10 & 4 & 5 & 16.979 & 41 & 14 & 15 & 154.021 & 72 & 24 & 23 & 434.625 \\
\hline 11 & 3 & 3 & 17.731 & 42 & 14 & 13 & 155.147 & 73 & 24 & 24 & 439.477 \\
\hline 12 & 4 & 3 & 19.933 & 43 & 14 & 14 & 159.835 & 74 & 25 & 26 & 469.607 \\
\hline 13 & 4 & 4 & 23.474 & 44 & 15 & 16 & 175.565 & 75 & 25 & 24 & 470.455 \\
\hline 14 & 5 & 6 & 24.316 & 45 & 15 & 14 & 176.641 & 76 & 25 & 25 & 475.303 \\
\hline 15 & 5 & 4 & 26.811 & 46 & 15 & 15 & 181.358 & 77 & 26 & 27 & 506.864 \\
\hline 16 & 5 & 5 & 30.653 & 47 & 16 & 17 & 198.538 & 78 & 26 & 25 & 507.693 \\
\hline 17 & 6 & 7 & 33.050 & 48 & 16 & 15 & 199.571 & 79 & 26 & 26 & 512.557 \\
\hline 18 & 6 & 5 & 35.211 & 49 & 16 & 16 & 204.314 & 80 & 27 & 28 & 545.547 \\
\hline 19 & 6 & 6 & 39.268 & 50 & 17 & 18 & 222.941 & 81 & 27 & 26 & 546.368 \\
\hline 20 & 7 & 8 & 43.193 & 51 & 17 & 16 & 223.938 & 82 & 27 & 27 & 551.238 \\
\hline 21 & 7 & 6 & 45.102 & 52 & 17 & 17 & 228.701 & 83 & 28 & 29 & 585.654 \\
\hline 22 & 7 & 7 & 49.318 & 53 & 18 & 19 & 248.774 & 84 & 28 & 27 & 586.367 \\
\hline 23 & 8 & 9 & 54.751 & 54 & 18 & 17 & 249.739 & 85 & 28 & 28 & 591.343 \\
\hline 24 & 8 & 7 & 56.467 & 55 & 18 & 18 & 254.521 & 86 & 29 & 30 & 627.187 \\
\hline 25 & 8 & 8 & 60.802 & 56 & 19 & 20 & 276.036 & 87 & 29 & 28 & 627.996 \\
\hline 26 & 9 & 10 & 67.731 & 57 & 19 & 18 & 276.974 & 88 & 29 & 29 & 632.874 \\
\hline 27 & 9 & 8 & 69.293 & 58 & 19 & 19 & 281.772 & 89 & 30 & 31 & 670.143 \\
\hline 28 & 9 & 9 & 73.722 & 59 & 20 & 21 & 304.727 & 90 & 30 & 29 & 670.947 \\
\hline 29 & 10 & 11 & 82.135 & 60 & 20 & 19 & 305.642 & 91 & 30 & 30 & 675.829 \\
\hline 30 & 10 & 9 & 83.574 & 61 & 20 & 20 & 310.454 & 92 & 31 & 32 & 714.521 \\
\hline 31 & 10 & 10 & 88.077 & 62 & 21 & 22 & 334.847 & 93 & 31 & 30 & 715.323 \\
\hline
\end{tabular}

minimum energy distance $\left(r_{\mathrm{e}}=2.80 \mathrm{bohr}\right)$. The ab initio surface was calculated using the MOLPRO package 2002. The three atoms were described by the aug-cc-pVQZ basis set (Woon \& Dunning 1994), augmented by the (3s3p2d2f1g) bond functions optimized by Cybulski \& Toczylowski (1999). Details of computations and plots of the PES are given in Paper I. For this van der Waals system, the global minimum of the interaction energy is found to be $-159.1127 \mu$ hartree i.e. $-34.921 \mathrm{~cm}^{-1}\left(R=7.29 a_{0}, \theta=180\right.$ degree $)$.

In the SO $\left({ }^{3} \Sigma^{-}\right)$electronic ground state, the rotational levels are split by spin-rotation coupling. In the intermediate coupling scheme, the rotational wave function of SO can be written for $j \geq 1$ as:

$$
\begin{aligned}
\left|F_{1} j m\right\rangle= & \cos \alpha|N=j-1, S j m\rangle \\
& +\sin \alpha|N=j+1, S j m\rangle \\
\left|F_{2} j m\right\rangle= & |N=j, S j m\rangle \\
\left|F_{3} j m\right\rangle= & -\sin \alpha|N=j-1, S j m\rangle \\
& +\cos \alpha|N=j+1, S j m\rangle
\end{aligned}
$$

where $|N, S j m\rangle$ denotes pure Hund's case (b) basis functions and the mixing angle $\alpha$ is obtained by diagonalisation of the molecular Hamiltonian. In the pure case (b) limit, $\alpha \rightarrow 0$, the $F_{1}$ level corresponds to $N=j-1$ and the $F_{3}$ level to $N=j+1$. For SO, the case (b) limit becomes valid beyond $N=5$. All calculations were carried out with the exact energy levels including the fine structure interaction. However, we will use in the present paper the usual level labeling $N_{j}$ where $N=j-1$, $N=j, N=j+1$ correspond to the $F_{1}, F_{2}$ and $F_{3}$ levels as defined in Eq. (1). The energies of the fine structure levels considered in the calculations were computed with the same effective Hamiltonian as in Paper I. The experimental spectroscopic constants of Bogey et al. (1982) were used. Energies of the 93 first fine structure levels are given in Table 1.

The detailed description of the CC calculations is given in Paper I. The quantal coupled equations were solved in the intermediate coupling scheme using the MOLSCAT code (Hutson $\&$ Green 1995) modified to take into account the fine structure of the energy levels. The propagation parameters were tested in order to obtain convergence of the cross sections. The calculations included all the open channels at each energy 
corresponding to energetically accessible levels. Several energetically inaccessible levels were included to obtain convergence of the collisional cross sections

Approximate cross sections among fine structure levels can be obtained in the Hund's case (b) limit using the IOS approximation (Corey \& Mc Court 1983):

$$
\begin{array}{r}
\sigma^{\mathrm{IOS}}\left(N S j \rightarrow N^{\prime} S j^{\prime}\right)=\sum_{L}(2 N+1)\left(2 N^{\prime}+1\right)\left(2 j^{\prime}+1\right) \\
\left(\begin{array}{rrr}
N^{\prime} & N & L \\
0 & 0 & 0
\end{array}\right)^{2}\left\{\begin{array}{ccc}
L & j & j^{\prime} \\
S & N^{\prime} & N
\end{array}\right\}^{2} \sigma^{\mathrm{IOS}}(0 \rightarrow L)
\end{array}
$$

where ( ) and \{ \} are respectively the "3-j" and "6-j" symbols.

$\sigma^{\mathrm{IOS}}(0 \rightarrow L)$ are the "fundamental IOS cross sections" obtained from the $L_{\mathrm{th}}$ expansion of the angle dependent S-matrix elements in terms of Legendre polynomials (Goldflam et al. 1977). As the IOS approximation neglects the energy structure of the levels, it is expected to be very poor near the level thresholds.

The way to obtain more reliable cross sections is to derive rates among fine structure levels from spin-free cross sections using the IOS approximation scaling relationship (hereafter IOSR) to recouple the angular momenta. This approximate recoupling method was described in detail by Neufeld \& Green (1994) for calculations of rate coefficients among hyperfine structure levels of $\mathrm{HCl}$ and used by Green (1994) for collisional excitation of $\mathrm{SO}$ by $\mathrm{H}_{2}$ molecules. In our case, we apply the scaling to downward cross sections:

$\sigma\left(N S j \rightarrow N^{\prime} S j^{\prime}\right)=\frac{\sigma^{\mathrm{IOS}}\left(N S j \rightarrow N^{\prime} S j^{\prime}\right)}{\sigma^{\mathrm{IOS}}\left(N \rightarrow N^{\prime}\right)} \sigma^{\mathrm{CC}}\left(N \rightarrow N^{\prime}\right)$

where:

$\sigma^{\mathrm{IOS}}\left(N \rightarrow N^{\prime}\right)=\sum_{L}\left(2 N^{\prime}+1\right)\left(\begin{array}{ccc}N^{\prime} & N & L \\ 0 & 0 & 0\end{array}\right)^{2} \sigma^{\mathrm{IOS}}(0 \rightarrow L)$

$\sigma^{\mathrm{IOS}}\left(N S j \rightarrow N^{\prime} S j^{\prime}\right)$ is given by Eq. (2) and $\sigma^{\mathrm{CC}}\left(N \rightarrow N^{\prime}\right)$ are the spin-free close coupling cross sections.

For the IOSR and IOS methods, the number of $L$ values considered in the summation is $0<L<N+N^{\prime}$ since the "3-j" and "6-j" coefficients are equal to 0 for the other $L$.

The MOLSCAT (Hutson \& Green 1995) code was also used for all the calculations with the IOS methods.

The downward rate coefficients are the Boltzmann thermal average of collision cross sections $\sigma_{u l}$ :

$k_{u l}(T)=\left(\frac{8 k_{\mathrm{B}} T}{\pi \mu}\right)^{\frac{1}{2}}\left(\frac{1}{k_{\mathrm{B}} T}\right)^{2} \times \int_{0}^{\infty} E_{\mathrm{k}} \sigma_{u l}\left(E_{\mathrm{k}}\right)^{\frac{-E_{\mathrm{k}}}{k_{\mathrm{B}} T}} \mathrm{~d} E_{\mathrm{k}}$

where $u$ and $l$ are respectively the upper and the lower states, $\mu$ is the reduced mass of the system, $E_{\mathrm{k}}$ is the kinetic energy and $k_{\mathrm{B}}$ is the Boltzmann constant.

The upward rate coefficients are obtained through detailed balance:

$k_{l u}=k_{u l} \frac{g_{u}}{g_{l}} \exp \left(-h v / k_{\mathrm{B}} T\right)$

where $g=2 j+1$ is the statistical weight.
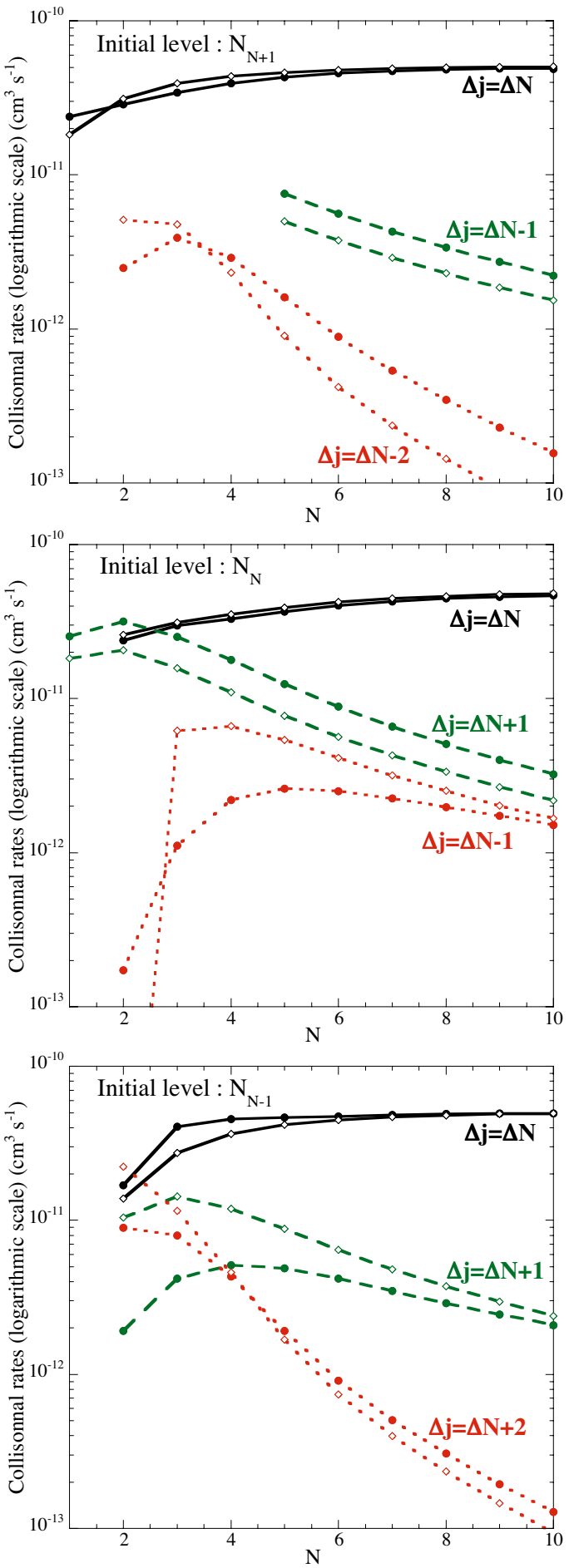

Fig. 1. CC rate coefficients (filled circles) compared with IOSR rate coefficients (empty diamonds) at $50 \mathrm{~K}$ for $\Delta N=N^{\prime}-N=-1$.

\section{Scattering calculations: comparison between CC and IOS methods}

As discussed in Paper I, the recoupling approximation IOSR is not expected to give accurate results due to the large fine structure splitting in SO. However, it is found to give a correct estimate of the rates among high $N$ levels and at high energy. Figure 1 compares the de-excitation $\mathrm{CC}$ rate coefficients with the approximate IOSR coefficients at $50 \mathrm{~K}$ for transitions 
$N_{j} \rightarrow N_{j^{\prime}}^{\prime}$ with $\Delta N=N^{\prime}-N=-1$. It is clear that the IOSR approximation is not valid among low lying levels $\left(N, N^{\prime}<5\right)$. However, when the upper level is higher than $N=5$, a good agreement is found for $\Delta j=\Delta N$ transitions. For transitions with $\Delta j \neq \Delta N$ (except $N_{N+1} \rightarrow N_{N^{\prime}-1}^{\prime}, \Delta j=\Delta N-2$ ), the difference between the methods decreases as $N$ increases and the IOSR approximation becomes reasonable. An important difference remains for the small rate coefficients of the $N_{N+1} \rightarrow N_{N^{\prime}-1}^{\prime}(\Delta j=\Delta N-2)$ transitions. This can be explained by the selection rules imposed by the $\{6 j\}$ coefficient in Eq. (2): the two first $L$ values corresponding to large $\sigma^{\mathrm{IOS}}(0 \rightarrow L)$ cross sections are excluded in the IOSR whereas such selection rules do not occur in full close coupling equations. However, as seen in Fig. 2, the difference becomes smaller with increasing energies so that we can expect to have a good agreement for the rate coefficients at high temperature $(T>100 \mathrm{~K})$. We also note in Fig. 1 the strong propensity rule $\Delta j=\Delta N$ transitions. This propensity rule, previously noted by Green (1994) for SO and by Corey et al. (1986) for $\mathrm{O}_{2}\left({ }^{3} \Sigma^{-}\right)-\mathrm{He}$ collisions, becomes stronger as $N$ increases.

The validity of the recoupling approximation IOSR compared to IOS is well analysed in Fig. 2 where a comparison between cross sections obtained with the CC, IOS and IOSR approaches is performed. For such cross sections between relatively high- $N$ levels, the agreement of IOSR results with the close coupling occurs at relatively low energy whereas the IOS approximation gives very poor results at low energies. This is due to a better description of the threshold in the IOSR approach. As expected, the difference between IOS and IOSR results decreases when the energy increases and we have found an excellent agreement between them at high energies. Rate coefficients were calculated using the energy grid and propagation parameters defined in Sect. 4.1. The coefficients obtained with the two methods are quite similar for temperature larger than $100 \mathrm{~K}$. This is due to the fact that spin-free CC and IOS cross sections are almost equal at high energies.

\section{Results}

\section{1. "Hybrid" cross sections and rate coefficients}

Cross sections were obtained for the 91 first levels of SO (see Table 1) and total energies up to $2000 \mathrm{~cm}^{-1}$ that allow us to obtain rate coefficients for temperature up to $300 \mathrm{~K}$. The reduced mass of the system is $3.694 \mathrm{amu}$. The propagation parameters were tested in order to obtain convergence of the cross sections for energies up to $2000 \mathrm{~cm}^{-1}$. Typically, the minimum and maximum integration distances are respectively $R_{\min }=2.75 a_{0}$ and $R_{\max }=40 a_{0}$. We carefully spanned the energy ranges to take into account the presence of resonances. The energy steps are $0.1 \mathrm{~cm}^{-1}$ below $30 \mathrm{~cm}^{-1}, 0.2 \mathrm{~cm}^{-1}$ from 30 to $50 \mathrm{~cm}^{-1}, 0.5 \mathrm{~cm}^{-1}$ from 50 to $100 \mathrm{~cm}^{-1}, 1 \mathrm{~cm}^{-1}$ from 200 to $800 \mathrm{~cm}^{-1}, 2 \mathrm{~cm}^{-1}$ from 800 to $1200 \mathrm{~cm}^{-1}, 4 \mathrm{~cm}^{-1}$ from 1200 to $2000 \mathrm{~cm}^{-1}$. According to the previous discussion, we used the following hybrid procedure: exact close coupling calculations were done for the 16 first levels $(N \leq 5)$ and total energies up to $2000 \mathrm{~cm}^{-1}$. The calculations included at each energy 43 levels in order to obtain convergence of the cross sections.
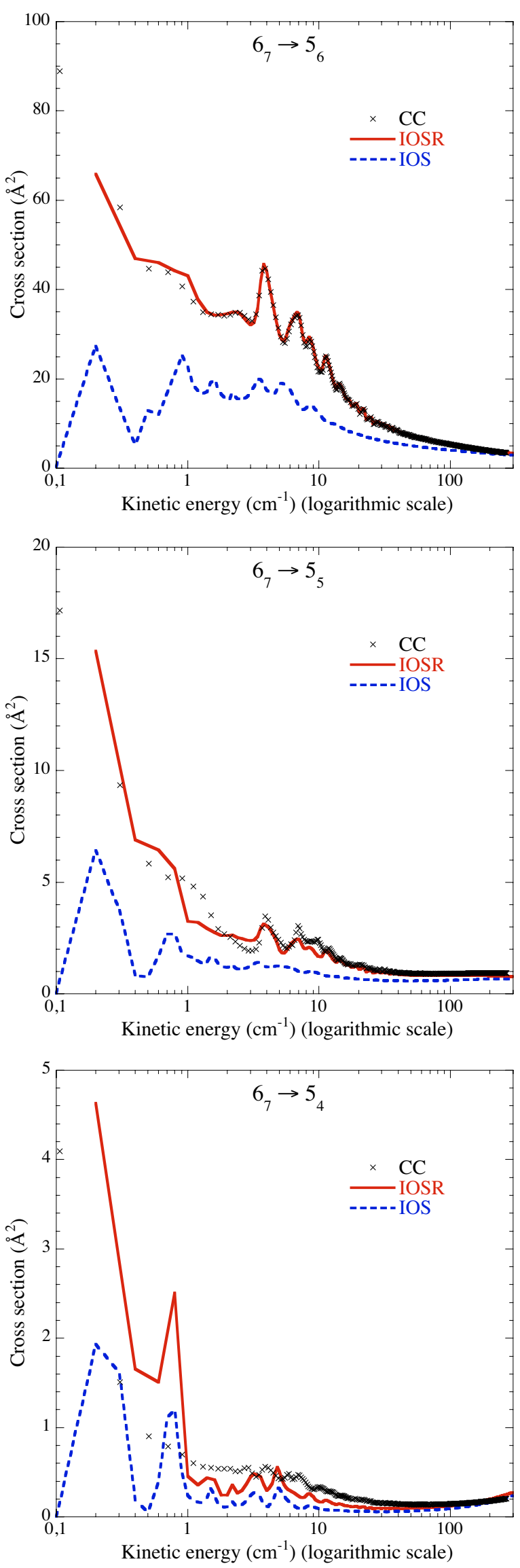

Fig. 2. Comparison between cross sections obtained with the CC, IOSR and IOS approaches. 
Table 2. Downward rate coefficients of fine structure levels of SO in collision with He as a function of kinetic temperature (in units of $\mathrm{cm}^{3} \mathrm{~s}^{-1}$ ).

\begin{tabular}{|c|c|c|c|c|c|c|c|c|c|c|c|c|c|}
\hline$N_{j}$ & & $\overline{N_{j^{\prime}}^{\prime}}$ & & $100 \mathrm{~K}$ & $200 \mathrm{~K}$ & $300 \mathrm{~K}$ & $\overline{N_{j}}$ & & $\overline{N_{j^{\prime}}^{\prime}}$ & & $100 \mathrm{~K}$ & $200 \mathrm{~K}$ & $300 \mathrm{~K}$ \\
\hline $0_{1}$ & & $1_{0}$ & & $1.47 \mathrm{e}-12$ & $1.05 \mathrm{e}-12$ & $9.84 \mathrm{e}-13$ & $4_{5}$ & & $1_{0}$ & & $1.74 \mathrm{e}-12$ & $2.11 \mathrm{e}-12$ & $2.21 \mathrm{e}-12$ \\
\hline $1_{2}$ & & $1_{0}$ & & $94 e-12$ & $02 \mathrm{e}-12$ & $09 e-12$ & $4_{5}$ & & $0_{1}$ & & 2 & 2 & $53 e-12$ \\
\hline $1_{2}$ & $\ldots$ & $0_{1}$ & & $13 e-11$ & $09 \mathrm{e}-11$ & $17 e-11$ & $4_{5}$ & $\cdots$ & $1_{2}$ & & $70 e-11$ & $.62 \mathrm{e}$ & $60 e-11$ \\
\hline 23 & $\cdots$ & $1_{0}$ & 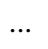 & & $5 e-$ & $7 e-12$ & $4_{5}$ & $\cdots$ & $2{ }_{3}$ & & $7 e-11$ & $73 e-11$ & $91 \mathrm{e}-11$ \\
\hline 23 & $\ldots$ & $0_{1}$ & $\ldots$ & $55 e-11$ & $.64 \mathrm{e}-11$ & $.74 \mathrm{e}-11$ & $4_{5}$ & $\ldots$ & $1_{1}$ & . & $7.62 \mathrm{e}-12$ & $20 \mathrm{e}-12$ & $.65 e-12$ \\
\hline 23 & 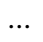 & $1_{2}$ & & $74 e-11$ & $84 \mathrm{e}-11$ & $2.99 \mathrm{e}-11$ & $4_{5}$ & $\cdots$ & $2{ }_{1}$ & & $3.98 \mathrm{e}-13$ & $.65 e-13$ & $.16 e-13$ \\
\hline $1_{1}$ &. & $1_{0}$ & & $43 e-13$ & $76 e-14$ & $3.50 \mathrm{e}-14$ & $4_{5}$ & $2+$ & $3_{4}$ & & $3.74 \mathrm{e}-11$ & $.80 \mathrm{e}-11$ & $.99 e-11$ \\
\hline $1_{1}$ & 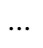 & $0_{1}$ & & $7 e-11$ & $2.60 \mathrm{e}-11$ & $2.79 \mathrm{e}-11$ & $4_{5}$ & & $2_{2}$ & & $1.44 \mathrm{e}-12$ & 2 & $82 \mathrm{e}-12$ \\
\hline $1_{1}$ & $\ldots$ & $1_{2}$ & $\ldots$ & & $8 e-11$ & $4.16 \mathrm{e}-11$ & $4_{5}$ & $\ldots$ & $3_{2}$ & & $3.90 \mathrm{e}-12$ & $e-12$ & $01 \mathrm{e}-12$ \\
\hline $1_{1}$ & $\ldots$ & 23 & $\ldots$ & -11 & $5 e-11$ & $02 \mathrm{e}-11$ & $3_{3}$ & ... & $1_{0}$ & 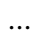 & $2.67 e-14$ & $.09 \mathrm{e}-14$ & $.52 \mathrm{e}-15$ \\
\hline $2{ }_{1}$ & 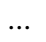 & $1_{0}$ & 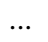 & -11 & $1 e-11$ & $84 \mathrm{e}-11$ & $3_{3}$ & . & $0_{1}$ & & $.75 e-11$ & -11 & $.54 \mathrm{e}-11$ \\
\hline 21 & $\ldots$ & $0_{1}$ & & $38 \mathrm{e}-12$ & $3.43 e-12$ & $3.59 \mathrm{e}-12$ & $3_{3}$ & & $1_{2}$ & & $5 e-11$ & 11 & $.51 \mathrm{e}-11$ \\
\hline $2{ }_{1}$ & ${ }^{2}$ & $1_{2}$ & te & & 2 & $e-12$ & $3_{3}$ & & 23 & & -11 & 11 & e-11 \\
\hline $2{ }_{1}$ & $\ldots$ & 23 & $\ldots$ & & $e-12$ & $79 e-12$ & $3_{3}$ & $\ldots$ & $1_{1}$ & $\cdots$ & $e-11$ & & $.70 e-11$ \\
\hline $2{ }_{1}$ & 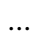 & $1_{1}$ & ... & 12 & $30 e-12$ & $33 e-12$ & $3_{3}$ & 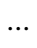 & $2{ }_{1}$ & & e-13 & & $.27 \mathrm{e}-13$ \\
\hline $3_{4}$ & & $1_{0}$ & & & 3 & -13 & $3_{3}$ & & 2 & & & & -11 \\
\hline $3_{4}$ & & $0_{1}$ & & & & -11 & & & & & & & -11 \\
\hline $3_{4}$ & $\ldots$ & $1_{2}$ & $\ldots$ & & $30 e-11$ & $46 e-11$ & $3_{3}$ & $\ldots$ & $3_{2}$ & & $5 e-12$ & -12 & e -12 \\
\hline $3_{4}$ & 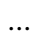 & 23 & & & $26 e-11$ & $44 e-11$ & $3_{3}$ & & $4_{5}$ & & $2.03 e-11$ & & $60 e-11$ \\
\hline $3_{4}$ & & $1_{1}$ & & 2 & $180 e-12$ & -12 & $4_{3}$ & & $1_{0}$ & & 11 & & Be-12 \\
\hline $3_{4}$ & & $2{ }_{1}$ & & 12 & $5.58 \mathrm{e}-$ & $e-12$ & $4_{3}$ & & $0_{1}$ & & -12 & & $2 e-12$ \\
\hline$z_{2}$ & $\cdots$ & $1_{0}$ & $\ldots$ & & $e-14$ & -14 & $4_{3}$ & ... & $1_{2}$ & $\cdots$ & -12 & & $e-12$ \\
\hline $22_{2}$ & 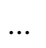 & $0_{1}$ & $\ldots$ & & 11 & -11 & $4_{3}$ & $\ldots$ & 23 & & 13 & & -12 \\
\hline $2{ }_{2}$ & & $1_{2}$ & & & 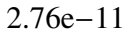 & $.75 e-11$ & $4_{3}$ & & $1_{1}$ & & & & $3 e-12$ \\
\hline $22_{2}$ & & 23 & & & $1.80 \mathrm{e}-11$ & $93 e-11$ & $4_{3}$ & & 2 & & 11 & & $.49 \mathrm{e}-11$ \\
\hline $22_{2}$ & $\cdots$ & $1_{1}$ & $\cdots$ & & & -11 & $4_{3}$ & $\cdots$ & $x_{4}+2$ & & $5.95 \mathrm{e}-12$ & 8.27 & $9.50 \mathrm{e}-12$ \\
\hline 22 & $\ldots$ & $2{ }_{1}$ & $\ldots$ & 12 & 1.24 & -12 & $4_{3}$ & 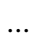 & $2_{2}$ & . & $2.47 \mathrm{e}-12$ & 2.56 & $2.73 e-12$ \\
\hline 22 & 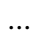 & $3_{4}$ & 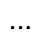 & & $3.20 \mathrm{e}-11$ & $3.27 \mathrm{e}-11$ & $4_{3}$ & & $3_{2}$ & & $4.54 \mathrm{e}-11$ & & $4.84 \mathrm{e}-11$ \\
\hline $3_{2}$ & $\ldots$ & $1_{0}$ & $\cdots$ & & $1.72 \mathrm{e}-11$ & $1.80 \mathrm{e}-11$ & $4_{3}$ & & $4_{5}$ & & $2.09 \mathrm{e}-12$ & 2.60 & $2.96 \mathrm{e}-12$ \\
\hline 2 & $\cdots$ & $0_{1}$ & $\cdots$ & & $3.02 \mathrm{e}-12$ & $2.89 \mathrm{e}-12$ & $4_{3}$ & $\cdots$ & 3 & & $5.33 \mathrm{e}-12$ & 5.61 & $5.71 \mathrm{e}-12$ \\
\hline $3_{2}$ & $\ldots$ & $1_{2}$ & $\ldots$ & & $1.58 \mathrm{e}-12$ & $1.70 \mathrm{e}-12$ & $4_{4}$ & .. & $1_{0}$ & & $1.21 \mathrm{e}-14$ & 4.87 & $2.86 e-15$ \\
\hline $3_{2}$ & ... & 23 & 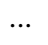 & & & $1.19 \mathrm{e}-11$ & $4_{4}$ & & $0_{1}$ & & $2.06 \mathrm{e}-12$ & & $2.32 \mathrm{e}-12$ \\
\hline $3_{2}$ & $\cdots$ & $1_{1}$ & ... & & $2.75 e-12$ & $2.93 e-12$ & $4_{4}$ & & $1_{2}$ & & $1.96 \mathrm{e}-11$ & 2.08 & $2.11 \mathrm{e}-11$ \\
\hline $3_{2}$ & $\cdots$ & $2_{1}$ & $\cdots$ & 11 & $3.67 \mathrm{e}-11$ & $3.76 \mathrm{e}-11$ & $4_{4}$ & ... & 2 & & $7.92 \mathrm{e}-12$ & 8.48 & $9.35 \mathrm{e}-12$ \\
\hline $3_{2}$ & . & $3_{4}$ & .. & & & & $+r$ & & & & & & $1.49 \mathrm{e}-11$ \\
\hline $3_{2}$ & $\cdots$ & $22_{2}$ & . & $3.60 \mathrm{e}-12$ & $3.30 \mathrm{e}-12$ & $3.25 \mathrm{e}-12$ & $4_{4}$ & $\ldots$ & $-L_{1}$ & $\cdots$ & $1.37 \mathrm{e}-13$ & $1.11 \mathrm{e}-13$ & $1.14 \mathrm{e}-13$ \\
\hline
\end{tabular}

For the next levels $(5<N \leq 15)$, the downward cross sections were calculated using the exact $\mathrm{CC}$ method for total energies up to $300 \mathrm{~cm}^{-1}$, the IOSR approximation (Eq. (3)) was used for energies between 300 and $700 \mathrm{~cm}^{-1}$ and the pure IOS approach (Eq. (2)) for energies up to $2000 \mathrm{~cm}^{-1}$. For the highest levels $(15<N \leq 30)$, the downward cross sections were calculated using the IOSR approach for total energies up to $700 \mathrm{~cm}^{-1}$ and the pure IOS for energies up to $2000 \mathrm{~cm}^{-1}$. With this method, we have described all the threshold energy ranges with a $\mathrm{CC}$ or a IOSR approach.

From this "hybrid treatment", we can evaluate the accuracy of the corresponding results. The first source of error comes from the PES. Recent investigations by Valiron (2005) permitted evaluation of the influence of the PES errors on the inelastic rates: accurate PES with few $\mathrm{cm}^{-1}$ accuracy such as the PES used in this work should lead to a 5-10\% accuracy in the rates. However, the main source of uncertainties comes from the scattering calculations. For all the levels with $N>5$, as explained above, it is clear that systematic errors result from the use of IOSR and IOS approximation instead of a full CC treatment.
The difference between IOSR and IOS for total energies larger than $700 \mathrm{~cm}^{-1}$ is very small and the errors are mainly due to the use of IOSR for total energies larger than $300 \mathrm{~cm}^{-1}$. For levels with $5<N \leq 15$, the difference between IOSR and CC cross sections at $300 \mathrm{~cm}^{-1}$ is smaller than $10 \%$ for $\Delta j=\Delta N$ transitions and smaller than $30-40 \%$ for $\Delta j \neq \Delta N$ transitions. For the highest levels ( $15<N \leq 30)$, the errors can be greater at low energies (up to a factor of 2) since the thresholds are not described correctly, but the difference decreases with increasing energies and is of the same order of magnitude as that found for the lowest levels at high energies. After a Boltzmann average, we can expect a reasonable error compared to a full CC calculations for all rate coefficients between levels with $5<N \leq 15$ : less than $5 \%$ for $\Delta j=\Delta N$ transitions, less than $20-30 \%$ for the small rate coefficients involving $\Delta j \neq \Delta N$ transitions. For the highest levels, the error can be up to $50 \%$ for low temperatures (50-100 K) but becomes similar to that of the lowest levels for higher temperatures.

The rate coefficients between the first $N$ levels are displayed in Tables 2 and 3 for temperatures equal to 100, 200 and $300 \mathrm{~K}$. 
Table 3. Downward rate coefficients of fine structure levels of SO in collision with $\mathrm{He}$ as a function of kinetic temperature (in units of $\mathrm{cm}^{3} \mathrm{~s}^{-1}$ ).

\begin{tabular}{|c|c|c|c|c|c|c|c|c|c|c|c|c|c|}
\hline$\overline{N_{j}}$ & & $\begin{array}{l}N_{j^{\prime}}^{\prime} \\
\end{array}$ & & $100 \mathrm{~K}$ & $200 \mathrm{~K}$ & $300 \mathrm{~K}$ & $\overline{N_{j}}$ & & $N_{j^{\prime}}^{\prime}$ & & 100 & 200 & 30 \\
\hline $44_{4}$ & & $3_{4}$ & & $03 \mathrm{e}-11$ & $33 \mathrm{e}-11$ & $2.48 \mathrm{e}-11$ & $5_{4}$ & $\cdots$ & $22_{1}$ & $\cdots$ & $2.23 \mathrm{e}-11$ & $2.24 \mathrm{e}-11$ & $2.23 \mathrm{e}-11$ \\
\hline $4_{4}$ & & $2_{2}$ & & $9 e-11$ & $18 \mathrm{e}-11$ & $.37 e-11$ & $5_{4}$ & & $3_{4}$ & & $33 e-12$ & $.64 \mathrm{e}-12$ & $85 e-12$ \\
\hline $4_{4}$ & & $3_{2}$ & . & 2 & 2 & $46 \mathrm{e}-$ & $5_{4}$ & & $22_{2}$ & & $65 e-12$ & & $e-12$ \\
\hline $4_{4}$ & . & $4_{5}$ & 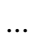 & & & $29 \mathrm{e}$ & $5_{4}$ & & & & $57 e-11$ & & $94 \mathrm{e}-11$ \\
\hline $4_{4}$ & $\ldots$ & $3_{3}$ & $\ldots$ & 1 & 11 & $63 e-11$ & $5_{4}$ & 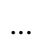 & $4_{5}$ & $\ldots$ & $06 e-12$ & -12 & $.47 \mathrm{e}-12$ \\
\hline $4_{4}$ & $\ldots$ & $4_{3}$ & $\ldots$ & $9 e-12$ & $64 e-12$ & $80 e-12$ & $5_{4}$ & & $3_{3}$ & $\ldots$ & $12 \mathrm{e}-12$ & $22 \mathrm{e}-12$ & $42 \mathrm{e}-12$ \\
\hline $5_{6}$ & 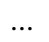 & $1_{0}$ & 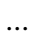 & Q & & $1.77 \mathrm{e}-$ & 5 & & 4 & & 11 & 11 & $13 e-11$ \\
\hline $5_{6}$ & .. & $0_{1}$ & & 6 & 12 & $8.04 \mathrm{e}-12$ & $5_{4}$ & & $4_{4}$ & & 12 & 12 & -12 \\
\hline $5_{6}$ & $\ldots$ & $1_{2}$ & $\ldots$ & & & $74 \mathrm{e}-12$ & $5_{4}$ & $\ldots$ & $5_{6}$ & $\ldots$ & & & $e-12$ \\
\hline $5_{6}$ & $\ldots$ & $2{ }_{3}$ & $\ldots$ & & & $2 \mathrm{e}-11$ & $5_{5}$ & $\ldots$ & $1_{0}$ & $\ldots$ & & & -15 \\
\hline $5_{6}$ & & $1_{1}$ & 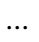 & $68 \mathrm{e}$ & 13 & $7.02 \mathrm{e}-13$ & $5_{5}$ & & $0_{1}$ & & $7 e-11$ & -11 & $1.30 \mathrm{e}-11$ \\
\hline $5_{6}$ & 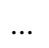 & $2{ }_{1}$ & & $3 \mathrm{e}$ & $54 \mathrm{e}-12$ & $4.06 \mathrm{e}-12$ & $5_{5}$ & & $1_{2}$ & & 12 & 12 & -12 \\
\hline $5_{6}$ & $\ldots$ & $3_{4}$ & $\ldots$ & 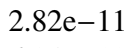 & & $3.35 \mathrm{e}$ & $5_{5}$ & & $2{ }_{3}$ & & 11 & & 11 \\
\hline $5_{6}$ & $\ldots$ & $2_{2}$ & $\ldots$ & 6 & 12 & $00 e-11$ & $5_{5}$ & $\ldots$ & $1_{1}$ & $\ldots$ & -12 & -12 & -12 \\
\hline $5_{6}$ & $\ldots$ & $3_{2}$ & $\ldots$ & 8 & 12 & $1.24 \mathrm{e}-12$ & $5_{5}$ & & $2{ }_{1}$ & & & & -13 \\
\hline $5_{6}$ & $\ldots$ & $4_{5}$ & & 4 & & $4.41 \mathrm{e}$ & $5_{5}$ & & & & 12 & & -12 \\
\hline $5_{6}$ & $\cdots$ & $3_{3}$ & & & & $2.35 \mathrm{e}$ & & & $L_{2}$ & & $1.73 \mathrm{e}-11$ & -11 & $1.76 \mathrm{e}-11$ \\
\hline $5_{6}$ & $\ldots$ & $4_{3}$ & $\ldots$ & 2. & 12 & $4.73 e-12$ & $5_{5}$ & $\ldots$ & $3_{2}$ & $\cdots$ & $2.22 \mathrm{e}-13$ & -13 & $2.62 \mathrm{e}-13$ \\
\hline $5_{6}$ & $\ldots$ & $4_{4}$ & . & & & -11 & $5_{5}$ & . & $4_{5}$ & . & & -11 & $1.95 \mathrm{e}-11$ \\
\hline $5_{4}$ & $\cdots$ & $1_{0}$ & $\cdots$ & & 12 & $1.26 \mathrm{e}-12$ & 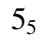 & & $3_{3}$ & & $2.49 \mathrm{e}-11$ & $2.69 \mathrm{e}-11$ & $2.89 \mathrm{e}-11$ \\
\hline $5_{4}$ & $\ldots$ & $0_{1}$ & $\cdots$ & & & $3.65 e-12$ & $5_{5}$ & $\cdots$ & 4 & & $2.98 \mathrm{e}-12$ & -12 & $3.66 e-12$ \\
\hline $5_{4}$ & $\ldots$ & $1_{2}$ & & & & & $5_{5}$ & & . & & & -11 & $3.92 \mathrm{e}-11$ \\
\hline $5_{4}$ & $\ldots$ & 23 & $\cdots$ & & & $6.24 \mathrm{e}-12$ & $5_{5}$ & $\cdot$ & $5_{6}$ & 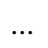 & & $6.31 \mathrm{e}-12$ & $7.42 \mathrm{e}-12$ \\
\hline $5_{4}$ & $\ldots$ & $1_{1}$ & $\ldots$ & $5.21 \mathrm{e}-13$ & $4.89 e-13$ & $5.28 \mathrm{e}-13$ & $5_{5}$ & $\ldots$ & $5_{4}$ & $\ldots$ & $1.57 \mathrm{e}-12$ & $1.70 \mathrm{e}-12$ & $1.88 \mathrm{e}-12$ \\
\hline
\end{tabular}

One observes a very slow variation of the coefficients with temperature. The results presented in this paper, the excitation rate coefficients for other temperatures and other transitions will be made available on our web site (Basecol).

\subsection{Comparison with previous work}

We compare our results to those of Green (1994), by transforming the $\mathrm{SO}-\mathrm{H}_{2}$ rates into $\mathrm{SO}-\mathrm{He}$ rates through the relation (Schöier et al. 2005):

$k_{\mathrm{SO}-\mathrm{He}}=k_{\mathrm{SO}-\mathrm{H}_{2}}\left(\frac{\mu_{\mathrm{SO}-\mathrm{H}_{2}}}{\mu_{\mathrm{SO}-\mathrm{He}}}\right)^{\frac{1}{2}}$.

Figure 3 compares rate coefficients for a number of transitions frequently observed at millimeter wavelengths. The difference is not a function of the temperature and does not change significantly when temperature increases. Large differences exist for the $0_{1} \rightarrow 1_{0}$ transition. They are due to the incorrect description of the energy levels in the approach of Green (1994) where fine structure is not taken into account. More generally, all downward transitions with $\Delta N>0$ should be described by a method that takes into account the exact fine structure splitting of the energy levels. For the other transitions with $\Delta j=\Delta N$, the differences are not so large and decrease when $\mathrm{N}$ increases. However, a difference of a factor of 2-3 is observed for the $6_{5} \rightarrow 5_{4}$ transition (Fig. 3) and more generally for all transitions described with the IOSR approach. We checked the accuracy of those rate coefficients against CC calculations; the difference is partially due to the use by Green (1994) of the PES calculated for the CS-He system with an electron gas model, modified at long range to smoothly join the asymptotic

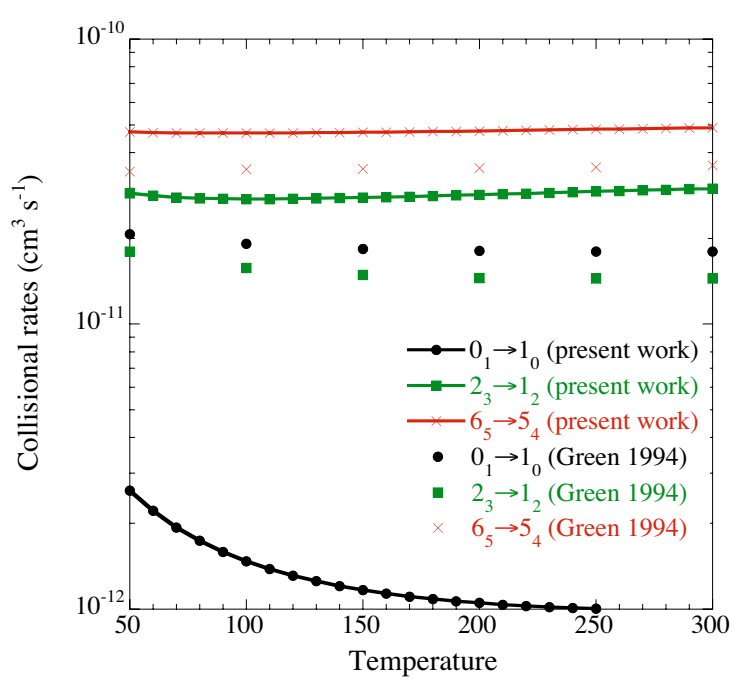

Fig. 3. Comparison between our rate coefficients (line + symbols) from 50 to $300 \mathrm{~K}$ with the results of Green (1994) (symbols).

interaction of CS with $\mathrm{H}_{2}$. As mentioned by Thomas et al. (1980), the electron gas model may lead to rates with error of up to a factor of 2-3, and Green pointed out that one might expect as much as a factor of 2-3 error for his calculated individual rate coefficients. The scaling factor given by Eq. (7) assumes that the cross section for $\mathrm{SO}-\mathrm{He}$ and $\mathrm{SO}-\mathrm{H}_{2}$ collisions are the same. Other than the differences in the PES, the difference in the reduced masses of the two colliding systems may contribute to different values of the cross sections. This will be tested in future work. 


\section{Conclusion}

We have extended into high temperature (50-300 K) our previous calculations obtained in the $\mathrm{CC}$ approach for low temperatures (Paper I). As a full CC cannot be used for high energies involving a very large number of open channels, we have defined a hybrid procedure combining the accurate $\mathrm{CC}$ approach to correctly define the first rotational levels with two approximate methods (IOS and IOS scaling relationship from spinfree cross sections). The final accuracy of the calculated rate coefficients is expected to be $5 \%$ for $\Delta j=\Delta N$ transitions and less than $20-30 \%$ for $\Delta j \neq \Delta N$ transitions compared to full CC calculations. A comparison with previous work by Green (1994) shows large differences, particularly for the first rotational levels. The consequences for astrophysical modeling are under study.

Acknowledgements. All the scattering calculations were performed at the IDRIS-CNRS center (Institut de Développement et des Ressources en Informatique Scientifique du Centre National de la Recherche Scientifique) under project 050883 and at the SIO of the observatory of Meudon.

\section{References}

Arthurs, A. M., \& Dalgarno, A. 1960, Proc. Roy. Soc. A, 256, 540 Blake, G. A., Surron, E. C., Masson, C. R., \& Phillips, T. G. 1987, ApJ, 315, 621

Basecol: http://boum. obspm. fr/basecol/

Bogey, M., Demuynck, C., \& Destombes, J. L. 1982, Chem. Phys., 66, 99
Boys, S. F., \& Bernardi, F. 1970, Mol. Phys., 19, 553

Corey, G. C., \& McCourt, F. R. 1983, J. Chem. Phys., 87, 2723

Corey, G. C., Alexander, M. H., \& Schaefer, J. 1986, J. Chem. Phys., 85,2726

Cybulski, S. M., \& Toczylowski, R. R. 1999, J. Chem. Phys., 111, 10520

Gottlieb, C. A., \& Ball, J. A. 1973, ApJ, 184, L59

Goldflam, R., Green, S., \& Kouri, D. J. 1977, J. Chem. Phys., 67, 4149, and references therein

Green, S. 1994, ApJ, 434, 188

Green, S., \& Chapman, S. 1978, ApJS, 37, 169

Hutson, J. M., \& Green, S. 1994, MOLSCAT computer code, version 14 (Collaborative Computational Project No. 6 of the Science and Engineering Research Council, UK)

Knowles, P. J., Hampel, C., \& Werner, H.-J. 1993, J. Chem. Phys., 99 5219

Knowles, P. J., Hampel, C., \& Werner, H.-J. 2000, J. Chem. Phys., 112,3106

Lique, F., Spielfiedel, A., Dubernet M.-L., \& Feautrier, N. 2005, J. Chem. Phys., 123, 134316

MOLPRO is a package of ab initio programs written by: Werner, H.-J., \& Knowles, P. J. with contributions from Almlöf, J., Amos, R. D., Deegan, M. J., et al.

Neufeld, D. A., \& Green, S. 1994, ApJ, 432, 158

Schöier, F. L., van der Tak, F. F. S., van Dishoeck, E. F., \& Black, J. H. 2005, A\&A, 432, 369

Thomas, L. D., Kraemer, W. P., \& Diercksen, G. H. F. 1980, Chem. Phys., 51, 131

Turner, B. E., Chan, K.-W., Green, S., \& Lubowich, D. A. 1992, ApJ, 399, 114

Valiron, P. 2005, personal communication

Van Dishoeck, E. F., \& Blake, G. A. 1998, ARA\&A, 36, 317

Woon, D. E., \& Dunning, Jr, T. H. 1994, J. Chem. Phys., 100, 2975 